REF - ISSN 1808-0804 Vol. VI (1), 25 - 32, 2009

\title{
DESENVOLVIMENTO E VALIDAÇÃO DE METODOLOGIA ANALÍTICA ESPECTROFOTOMÉTRICA PARA DOSEAMENTO DE MEBENDAZOL MATÉRIA-PRIMA E SUSPENSÃO ORAL
}

\author{
Development and validation and of spectrophotometrical analytical methodology for \\ quantification of mebendazole raw material and oral suspension
Ezequiane Machadom Silva ; Lívia Teixeira Duarte ; Andrezza Lopes Sousa; Daniella Ramos Martins; Larissa da Cunha Almeida;
Paula Izabella Rocha de Magalhães Pereira; Clévia Ferreira Duarte Garrote; Maria Teresa Freitas Bara*

\footnotetext{
${ }^{1}$ Laboratório de Controle de Qualidade de Medicamentos (LCQM), Faculdade de Farmácia; Universidade Federal de Goiás, Av. Universitária com 1ํAvenida s/n, Setor Universitário CEP: 74605-220 - Goiânia - GO. Brasil.
}

*Autor para correspondência e-mail: mbara@farmacia.ufg.br

Recebido em 15/05/2008 - Aceito em 22/12/2008

RESUMO: O mebendazol é um fármaco que atua como anti-helmíntico de amplo espectro de ação contra nematóides e cestóides. Entre as metodologias utilizadas para sua determinação, destaca-se a possibilidade de determinação quantitativa de mebendazol por meio de métodos espectrofotométricos, por apresentarem boa sensibilidade e ter custos mais acessíveis. O presente trabalho teve como objetivo desenvolver e validar uma metodologia analítica para determinação deste fármaco em matéria-prima e suspensão oral, utilizando a espectrofotometria de ultravioleta. O método mostrou ser simples, rápido, específico, linear, exato, preciso e robusto para ser executado na rotina de um laboratório de controle de qualidade, sendo uma alternativa aos métodos utilizados.

PALAVRAS-CHAVE: controle de qualidade, análise farmacêutica, espectrofotometria.

ABSTRACT: The mebendazole is a drug that acts as anti-helminthic of broad spectrum of action against nematodes and cestodes. Among the methodologies used for their determination, highlights the possibility for quantitative determination of mebendazole is through methods spectrophotometrics, to have good sensitivity and have cost more accessible. This study aimed to develop and validate a method for analytical determination of mebendazole in raw material and in oral suspension, using the ultraviolet spectrophotometry. The method proved to be simple, rapid, specific, linear, accurate, precise and robust to run the routine of a laboratory of quality control, and is an alternative to methods used.

KEYWORDS: quality control, pharmaceutical analysis, spectrophotometry.

\section{INTRODUÇÃO}

O mebendazol é um derivado benzimidazólico (Figura 1) utilizado como anti-helmíntico com elevada eficácia e tolerabilidade, além de um amplo espectro de ação contra nematóides e cestóides (KATZUNG, 1998;

SILVA, 2006). 
<smiles>COC(=O)Nc1nc2cc(C(=O)c3ccccc3)ccc2[nH]1</smiles>

Figura 1 - Estrutura molecular do mebendazol

Dentre as atribuições de um laboratório de controle de qualidade destaca-se a garantia de que sejam feitas as validações necessárias, de procedimentos analíticos, calibração e qualificação dos equipamentos e vidrarias de precisão (LACHMAN et al., 2001; PINTO et al, 2003).

Uma validação visa demonstrar que o método é apropriado para a finalidade pretendida, garantindo por meio de estudos experimentais, que o método atenda aos parâmetros de especificidade, linearidade, intervalo, precisão, limite de detecção, limite de quantificação, exatidão e robustez adequados à análise (BRASIL, 2003).

A monografia do mebendazol - matéria-prima está presente na Farmacopéia Brasileira 4⿳a edição (2001), na qual o doseamento é feito por titulação em meio não aquoso. No entanto, considera-se que o método titulométrico possui menor sensibilidade e pode sofrer maior número de interferências quando comparado ao método espectrofotométrico. A Farmacopéia Brasileira 4aํ Edição, 2005 descreve duas técnicas analíticas por espectrofotometria para suspensão oral de mebendazol, porém uma desta utiliza concentrações muito altas de ácido fórmico, o que a torna insalubre e de alta periculosidade para o analista. A outra técnica tem como desvantagem o uso de solventes orgânicos como isopropanol e clorofórmio (FARMACOPÉIA BRASILEIRA, 2005). Além disso, ambas possuem etapa de extração líquido-líquido, que aumenta a possibilidade de interferências.

Este trabalho visou o desenvolvimento e validação de uma metodologia espectrofotométrica para o doseamento de mebendazol em matérias-primas e em suspensão oral, que seja uma alternativa mais viável à rotina de um laboratório de controle de qualidade.

\section{MÉTODOS}

\section{Material e equipamento}

Foram utilizadas mebendazol SQR (Farmacopéia Brasileira), mebendazol - matéria-prima e suspensão oral de mebendazol manipulada em farmácia com manipulação.

Os solventes utilizados possuiam grau analítico e foram utilizados ácido fórmico $99 \%$ (Vetec) e etanol absoluto grau UV/HPLC (JT Baker).

A vidraria utilizada foi certificada pela RBC (Rede Brasileira de Calibração).

\section{Doseamento de Mebendazol matéria-prima e suspensão oral}

\section{Preparo do padrão:}

Transferir $25 \mathrm{mg}$ de padrão secundário de mebendazol para balão volumétrico de $25 \mathrm{~mL}$.

Acrescentar $12 \mathrm{~mL}$ de ácido fórmico P.A. $(99,0 \%)$ e solubilizar com agitação e/ou banho de ultrassom. Completar o volume com etanol P.A.

Transferir $1 \mathrm{~mL}$ desta solução para balão volumétrico de $100 \mathrm{~mL}$ e completar o volume com etanol P.A. Esta solução contém $0,01 \mathrm{mg} / \mathrm{mL}$.

\section{Preparo da amostra}

Transferir o equivalente a $100 \mathrm{mg}$ de mebendazol para balão volumétrico de $100 \mathrm{~mL}$.

Acrescentar $12 \mathrm{~mL}$ de ácido fórmico P.A. $(99,0 \%)$ e solubilizar com agitação e/ou banho de ultrassom. Completar o volume com etanol P.A. Transferir $1 \mathrm{~mL}$ desta solução para balão volumétrico de $100 \mathrm{~mL} e$ completar o volume com etanol P.A.

Medir as absorbâncias das soluções resultantes (padrão e amostra) em 310nm, utilizando etanol para o ajuste do zero.

Calcular o teor de mebendazol na amostra a partir das leituras obtidas. 
Onde:

$$
\mathrm{T}=\frac{\mathrm{Cp} \times \mathrm{Aa} \times 100}{\operatorname{Cta} \times A p}
$$

$\mathrm{T}=$ teor da amostra em porcentagem;

$\mathrm{Cp}=$ concentração do padrão $(\mathrm{mg} / \mathrm{mL})$;

$\mathrm{Aa}=$ absorbância da amostra;

Cta = concentração teórica da amostra;

$\mathrm{Ap}=$ absorbância do padrão.

Foram utilizados espectrofotômetros (B582/Micronal e Cary 50/Varian) e comprimento de onda de leitura de $310 \mathrm{~nm}$. O ajuste do zero foi feito com etanol absoluto.

No estudo da validação do método para matéria-prima foram avaliados os parâmetros: especificidade, linearidade, precisão, limite de quantificação, limite de detecção, exatidão e robustez.

Os parâmetros avaliados na validação do método para o produto-acabado foram : especificidade, linearidade, intervalo, precisão (repetibilidade e precisão intermediária), exatidão e robustez.

\section{RESULTADOS E DISCUSSÃo}

O desenvolvimento de métodos analíticos empregados na avaliação de qualidade é de fundamental importância e colabora com as Boas Práticas de Fabricação, integrando os procedimentos relacionados com a Garantia da Qualidade (GIL, 2007).

Todo método desenvolvido e não descrito em farmacopéia ou formulários oficiais deve ser validado, sendo avaliados os seguintes parâmetros: especificidade, linearidade, intervalo, precisão, exatidão e robustez, quando se trata da quantificação do analito em formas farmacêuticas. Para este fim, outros documentos podem ser empregados, além do guia da ANVISA (BRASIL, 2003), tais como United States Pharmacopoea - USP (2007), International Conference on Harmonisation - ICH Q2, (2005), Food and Drug Administration - FDA (1994), Instituto Nacional de Metrologia - INMETRO (2003), visando uma validação.

O desenvolvimento da metodologia analítica para substituir a análise titrimétrica constante na monografia da Farmacopéia Brasileira (2001) baseou-se inicialmente na solubilidade do fármaco em ácido fórmico e etanol (Farmacopéia Brasileira, 2005). Para tanto, demonstrou-se inicialmente a especificidade do método empregado na análise da matéria-prima, uma vez que o mesmo não sofreu interferência do solvente utilizado no comprimento de onda de detecção (310nm). Os espectros da varredura das soluções padrão e amostra foram idênticos, já o espectro da varredura do solvente não apresentou nenhum pico de absorção (Figura 2).
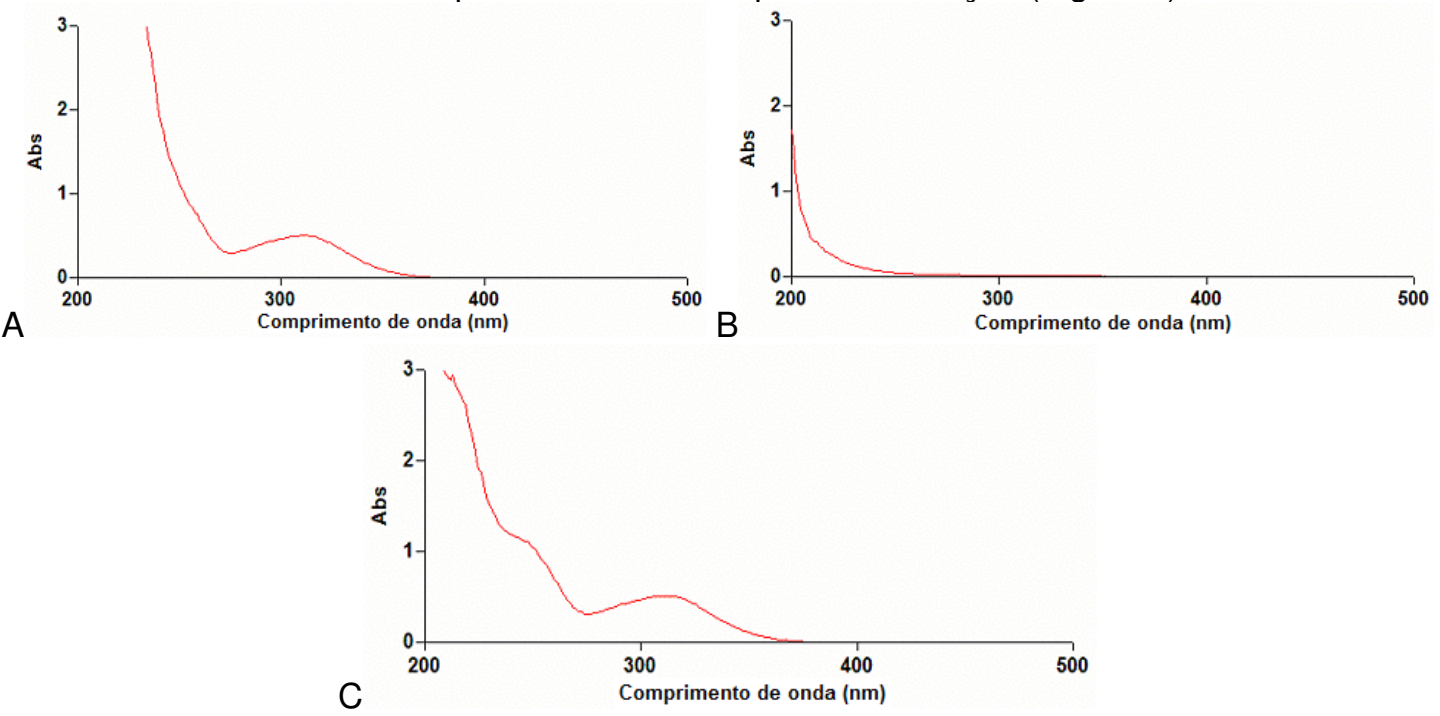

Figura 2 - Espectros de absorção do padrão (A), solvente (B) e amostra (C), respectivamente.

Os resultados para o parâmetro de linearidade (Figura 3) demonstraram que o método apresenta intervalo linear na faixa de 50 a $150 \%$. 


\section{Curva de calibração mebendazol}

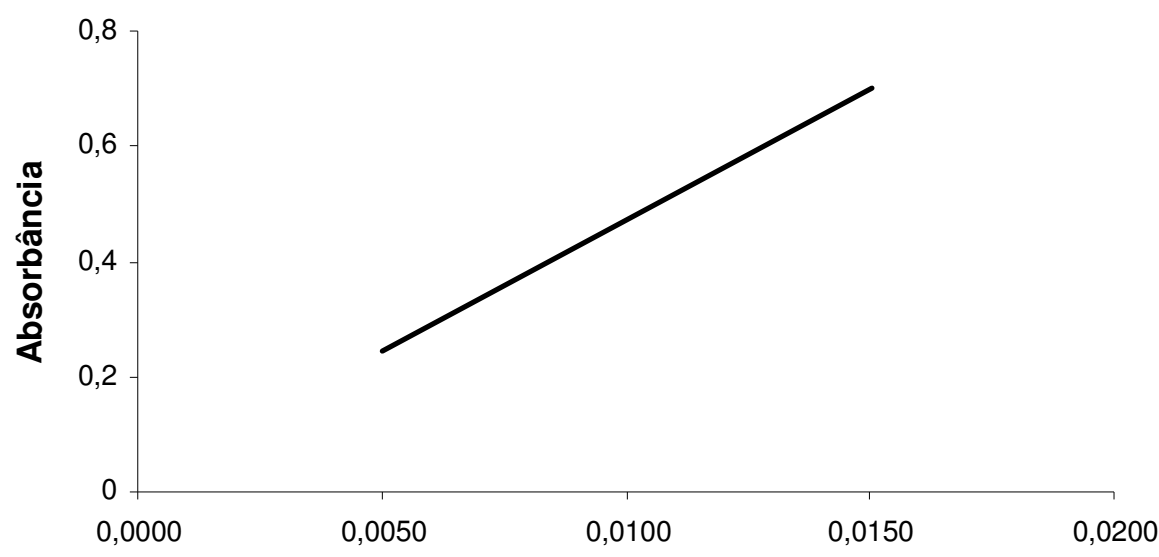

Concentração da Solução Padrão de Mebendazol (mg/mL)

Figura 3 - Linearidade: $\quad y=45,155 x+0,0186 \quad r=0,99989$

O método apresentou-se preciso nos dois níveis avaliados (Tabelas 1 e 2), com coeficientes de variação (CV) inferiores ao especificado pela resolução vigente $(5,0 \%), 0,82$ e 1,3\%, respectivamente para repetibilidade e precisão intermediária.

Tabela 1 - Resultados da Precisão (repetibilidade)

\begin{tabular}{|c|c|c|c|c|c|}
\hline Determinações & $\begin{array}{c}\text { Massas } \\
\text { pesadas } \\
(\mathrm{mg})\end{array}$ & $\begin{array}{c}\text { Concentração } \\
\text { teórica da } \\
\text { amostra } \\
(\mathrm{mg} / \mathrm{mL})\end{array}$ & $\begin{array}{c}\text { Concentração } \\
\text { real da } \\
\text { amostra } \\
(\mathrm{mg} / \mathrm{mL})\end{array}$ & $\begin{array}{c}\text { Recuperação } \\
(\%)\end{array}$ & Absorbâncias \\
\hline D_1 & 100,2 & 0,01002 & 0,01019 & 101,73 & 0,4789 \\
\hline$D_{2} 2$ & 100,3 & 0,01003 & 0,01035 & 103,20 & 0,4860 \\
\hline D 3 & 100,8 & 0,01008 & 0,01038 & 102,95 & 0,4872 \\
\hline$D^{-4} 4$ & 100,4 & 0,01004 & 0,01026 & 102,19 & 0,4819 \\
\hline D 5 & 100,5 & 0,01005 & 0,01027 & 102,22 & 0,4825 \\
\hline \multirow{7}{*}{ D_6 } & 100,5 & 0,01005 & 0,01014 & 100,88 & 0,4764 \\
\hline & & & & Média & \\
\hline & & & & 102,20 & \\
\hline & & & & DP & \\
\hline & & & & 0,839945672 & \\
\hline & & & & DPR & \\
\hline & & & & 0,821885964 & \\
\hline
\end{tabular}

$\mathrm{DP}=$ desvio padrão $\quad$ DPR $=$ desvio padrão relativo

Tabela 2 - Resultados da Precisão (intermediária)

\begin{tabular}{cccccc}
\hline Determinações & $\begin{array}{c}\text { Massas } \\
\text { pesadas } \\
(\mathrm{mg})\end{array}$ & $\begin{array}{c}\text { Concentração } \\
\text { teórica da } \\
\text { amostra } \\
(\mathrm{mg} / \mathrm{mL})\end{array}$ & $\begin{array}{c}\text { Concentração } \\
\text { real da } \\
\text { amostra } \\
(\mathrm{mg} / \mathrm{mL})\end{array}$ & Teor $(\%)$ & Absorbâncias \\
\hline D_1 & 100,1 & 0,01001 & 0,01006 & 100,49 & 0,4728 \\
D_2 & 100,2 & 0,01002 & 0,01027 & 102,51 & 0,4824 \\
D_3 & 100,3 & 0,01003 & 0,01030 & 102,71 & 0,4838 \\
D_4 & 100,2 & 0,01002 & 0,01019 & 101,73 & 0,4789 \\
D_5 & 100,0 & 0,01000 & 0,01030 & 103,00 & 0,4837
\end{tabular}




\begin{tabular}{c} 
Média \\
\hline 101,70 \\
\hline DP \\
\hline 1,320066686 \\
\hline DPR \\
\hline 1,298046905
\end{tabular}

$\mathrm{DP}=$ desvio padrão

DPR $=$ desvio padrão relativo

A exatidão do método foi outro parâmetro comprovado, sendo encontrados valores entre 97,38 e 103,88 para as três concentrações investigadas (Tabela 3).

Tabela 3 - Resultados da Exatidão

\begin{tabular}{cccccccccc}
\hline $\begin{array}{c}\text { Determi- } \\
\text { nações }\end{array}$ & $\begin{array}{c}\text { Massas } \\
\text { pesadas } \\
(\mathrm{mg})\end{array}$ & $\begin{array}{c}\text { Concentração } \\
\text { teórica da } \\
\text { amostra } \\
(\mathrm{mg} / \mathrm{mL})\end{array}$ & $\begin{array}{c}\text { Concentração } \\
\text { real da } \\
\text { amostra } \\
(\mathrm{mg} / \mathrm{mL})\end{array}$ & $\begin{array}{c}\text { Recupe- } \\
\text { ração } \\
(\%)\end{array}$ & $\begin{array}{c}\text { Absorbân- } \\
\text { cias }\end{array}$ & $\begin{array}{c}\text { Média } \\
\text { Recupe- } \\
\text { ração } \\
(\%)\end{array}$ & DP & DPR & $\begin{array}{c}\text { Recuperação } \\
(\mathrm{mg} / \mathrm{mL})\end{array}$ \\
\hline D_1 & 50,1 & 0,00501 & 0,00486 & 96,98 & 0,2380 & & & & 0,00485882 \\
D_2 & 50,0 & 0,00500 & 0,00491 & 98,28 & 0,2405 & 97,3867 & 0,7781 & 0,80 & 0,004914184 \\
D_3 & 50,1 & 0,00501 & 0,00485 & 96,89 & 0,2378 & & & & 0,00485439 \\
D_4 & 100,2 & 0,01002 & 0,01020 & 101,76 & 0,4790 & & & & 0,010195992 \\
D_5 & 100,3 & 0,01003 & 0,01036 & 103,24 & 0,4862 & 102,7610 & 0,8702 & 0,85 & 0,010355442 \\
D_6 & 100,8 & 0,01008 & 0,01041 & 103,28 & 0,4887 & & & & 0,010410807 \\
D_7 & 150,2 & 0,01502 & 0,01562 & 104,01 & 0,7240 & & & & 0,015621747 \\
D_8 & 150,2 & 0,01502 & 0,01569 & 104,48 & 0,7272 & 103,88 & 0,6779 & 0,65 & 0,015692614 \\
D_9 & 150,3 & 0,01503 & 0,01550 & 103,14 & 0,7186 & & & & 0,015502159 \\
\hline DP
\end{tabular}

Os limites de detecção $\left(6,63 \times 10^{-5} \mathrm{mg} / \mathrm{mL}\right)$ e de quantificação $\left(2,21 \times 10^{-5} \mathrm{mg} / \mathrm{mL}\right)$ obtidos demonstram que o método é sensível.

O método foi robusto para variações de até 2 unidades no comprimento de onda e para variações da marca do espectrofotômetro. Os coeficientes de variação obtidos estão dentro dos limites preconizados (Tabela 4).

Tabela 4 - Resultados da robustez

\begin{tabular}{l|c|c|c|c|c|c}
\hline & Absorvância & Média & $\begin{array}{c}\text { Concentração } \\
\text { real da } \\
\text { amostra } \\
\text { Parâmetro }\end{array}$ & DP & DPR \\
\hline \multirow{2}{*}{$\lambda(\mathrm{mm})$} & 308 & 0,4765 & 0,477367 & 0,01016 & 0,005551 & $\begin{array}{c}1,16283 \\
3\end{array}$ \\
\cline { 2 - 4 } & 310 & 0,4833 & & & & \\
\hline
\end{tabular}




\begin{tabular}{|c|c|c|c|c|c|c|}
\hline & 312 & 0,4723 & & & & \\
\hline \multirow{2}{*}{ Equipamento } & 1 & 0,4833 & \multirow{2}{*}{0,479650} & \multirow{2}{*}{0,01021} & \multirow{2}{*}{0,005162} & \multirow{2}{*}{$\begin{array}{c}1,07617 \\
6\end{array}$} \\
\hline & 2 & 0,4760 & & & & \\
\hline
\end{tabular}

$\overline{\mathrm{DP}}=$ desvio padrão $\quad$ DPR $=$ desvio padrão relativo

O método quando empregado para análise da suspensão oral de mebendazol apresentou-se específico, pois foram observados picos de absorbância no comprimento de onda $310 \mathrm{~nm}$ para o padrão e a amostra, não sendo observado pico significativo na varredura da matriz (Figura 4).

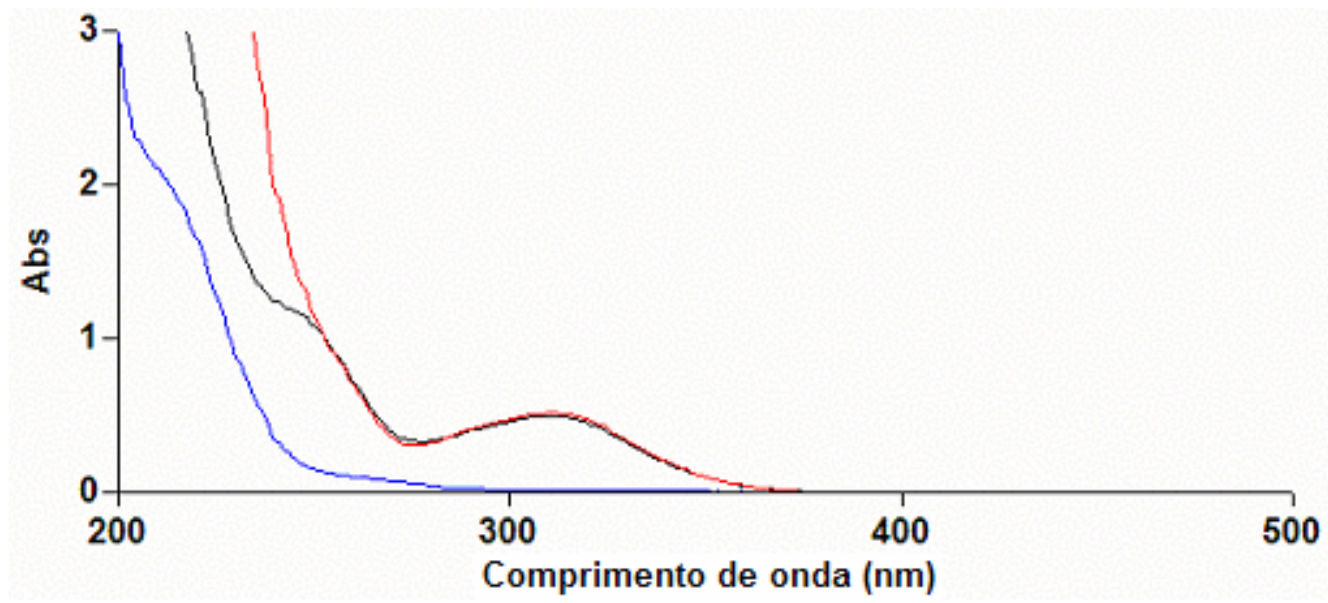

Figura 4: Espectros de absorção - Matriz (azul), amostra (preto) padrão (vermelho) de mebendazol

O intervalo linear para a análise da suspensão de mebendazol foi estabelecido entre as concentrações de $0,005 \mathrm{mg} / \mathrm{mL}$ a $0,0151 \mathrm{mg} / \mathrm{mL}$ (Figura 2).

O método proposto neste estudo demonstrou precisão e obteve-se um DPR de 2,27 para a repetibilidade e 2,12 para a precisão intermediária (Tabelas 5 e 6).

Tabela 5 - Resultados da Precisão (repetibilidade) - suspensão oral

\begin{tabular}{cccccccc}
\hline Determinações & $\begin{array}{c}\text { Massas } \\
\text { pesadas } \\
(\mathrm{g})^{*}\end{array}$ & $\begin{array}{c}\text { Volume } \\
\text { equivalente } \\
(\mathrm{mL})\end{array}$ & $\begin{array}{c}\text { Equivalente } \\
\text { em mg }\end{array}$ & $\begin{array}{c}\text { Concentração } \\
\text { teórica da } \\
\text { amostra } \\
(\mathrm{mg} / \mathrm{mL})\end{array}$ & $\begin{array}{c}\text { Concentração } \\
\text { real da } \\
\text { amostra } \\
(\mathrm{mg} / \mathrm{mL})\end{array}$ & $\begin{array}{c}\text { Recupera- } \\
\text { ção }(\%)\end{array}$ & $\begin{array}{c}\text { Absorbân- } \\
\text { cias }\end{array}$ \\
\hline D_1 & 6,3880 & 5,027546041 & 100,5509208 & 0,01006 & 0,01032 & 102,63 & 0,4846 \\
D_2 & 6,3447 & 4,993467653 & 99,86935306 & 0,00999 & 0,01055 & 105,66 & 0,4951 \\
D_3 & 6,3874 & 5,027073823 & 100,5414765 & 0,01005 & 0,01000 & 99,45 & 0,4701 \\
D_4 & 6,3404 & 4,990083425 & 99,8016685 & 0,00998 & 0,01008 & 101,03 & 0,4739 \\
D_5 & 6,3832 & 5,023768298 & 100,475366 & 0,01005 & 0,01006 & 100,09 & 0,4727 \\
D_6 & 6,3422 & 4,991500079 & 99,83000157 & 0,00998 & 0,01000 & 100,20 & 0,4703 \\
\hline
\end{tabular}

$\frac{\frac{\text { Média }}{\mathrm{DP}}}{2,311435766}$


${ }^{*}$ Densidade $(\mathrm{g} / \mathrm{mL}): 1,270597612 . \quad \mathrm{DP}=$ desvio padrão $\quad$ DPR= desvio padrão relativo A densidade foi empregada para transformar a massa pesada em volume da suspensão oral.

Tabela 6 - Resultados da Precisão (intermediária) - suspensão oral

\begin{tabular}{cccccccc}
\hline Determinações & $\begin{array}{c}\text { Massas } \\
\text { pesadas } \\
(\mathrm{g})^{*}\end{array}$ & $\begin{array}{c}\text { Volume } \\
\text { equivalente } \\
(\mathrm{mL})\end{array}$ & $\begin{array}{c}\text { Equivalente } \\
\text { em mg }\end{array}$ & $\begin{array}{c}\text { Concentração } \\
\text { teórica da } \\
\text { amostra } \\
(\mathrm{mg} / \mathrm{mL})\end{array}$ & $\begin{array}{c}\text { Concentração } \\
\text { real da } \\
\text { amostra } \\
(\mathrm{mg} / \mathrm{mL})\end{array}$ & $\begin{array}{c}\text { Recupera- } \\
\text { ção }(\%)\end{array}$ & $\begin{array}{c}\text { Absorbân- } \\
\text { cias }\end{array}$ \\
\hline D_1 & 6,5088 & 5,122619235 & 102,4523847 & 0,01025 & 0,00991 & 96,73 & 0,4661 \\
D_2 & 6,5643 & 5,166299386 & 103,3259877 & 0,01033 & 0,01023 & 99,00 & 0,4805 \\
D_3 & 6,4936 & 5,110656383 & 102,2131277 & 0,01022 & 0,01031 & 100,88 & 0,4842 \\
D_4 & 6,4741 & 5,095309303 & 101,9061861 & 0,01019 & 0,01018 & 99,86 & 0,4781 \\
D_5 & 6,4109 & 5,045569023 & 100,9113805 & 0,01009 & 0,01029 & 101,94 & 0,4831 \\
D_6 & 6,4099 & 5,044781993 & 100,8956399 & 0,01009 & 0,01035 & 102,57 & 0,4859 \\
\hline
\end{tabular}

\begin{tabular}{|c|}
\hline Média \\
\hline DP \\
\hline 2,12995894 \\
\hline DPR \\
\hline 2,126505067 \\
\hline
\end{tabular}

${ }^{*}$ Densidade $(\mathrm{g} / \mathrm{mL}): 1,270597612 . \quad \mathrm{DP}=$ desvio padrão $\quad \mathrm{DPR}=$ desvio padrão relativo

A densidade foi empregada para transformar a massa pesada em volume da suspensão oral.

A exatidão do método foi comprovada através dos percentuais de recuperação encontrados entre 95,71 e 100,69\% nas três concentrações analisadas (Tabela 7).

Tabela 7 - Resultados da exatidão - suspensão oral

\begin{tabular}{|c|c|c|c|c|c|c|c|c|c|}
\hline $\begin{array}{l}\text { Determi- } \\
\text { nações }\end{array}$ & $\begin{array}{l}\text { Massas } \\
\text { pesadas } \\
\text { (g) }\end{array}$ & $\begin{array}{l}\text { Volume } \\
\text { equivalente } \\
(\mathrm{mL})\end{array}$ & $\begin{array}{l}\text { Equiva- } \\
\text { lente } \\
\text { (mg) }\end{array}$ & $\begin{array}{l}\text { Concentração } \\
\text { teórica da } \\
\text { amostra } \\
(\mathrm{mg} / \mathrm{mL})\end{array}$ & $\begin{array}{c}\text { Concentração } \\
\text { real da } \\
\text { amostra } \\
(\mathrm{mg} / \mathrm{mL})\end{array}$ & $\begin{array}{l}\text { Recupe- } \\
\text { ração } \\
(\%)\end{array}$ & $\begin{array}{l}\text { Absor- } \\
\text { bâncias }\end{array}$ & $\begin{array}{c}\text { Média } \\
\text { Recupe- } \\
\text { ração (\%) }\end{array}$ & DP \\
\hline D_1 & 3,1900 & 2,48055988 & 49,6112 & 0,00496 & 0,00472 & 95,17 & 0,2318 & & \\
\hline D_2 & 3,3543 & 2,60832037 & 52,1664 & 0,00522 & 0,00507 & 97,13 & 0,2474 & 95,709 & 1,2440 \\
\hline D_3 & 3,1791 & 2,47208398 & 49,4417 & 0,00494 & 0,00469 & 94,82 & 0,2303 & & \\
\hline D_4 & 6,3874 & 5,02707382 & 100,5415 & 0,01005 & 0,00999 & 99,36 & 0,4697 & & \\
\hline D_5 & 6,3404 & 4,99008343 & 99,8017 & 0,00998 & 0,01006 & 100,79 & 0,4728 & 100,095 & 0,7131 \\
\hline D_6 & 6,3422 & 4,99150008 & 99,8300 & 0,00998 & 0,01000 & 100,14 & 0,4700 & & \\
\hline D_7 & 5,8861 & 5,05157913 & 151,5474 & 0,01515 & 0,01543 & 101,80 & 0,7152 & & \\
\hline D_8 & 5,8761 & 5,04299691 & 151,2899 & 0,01513 & 0,01500 & 99,17 & 0,6961 & 100,69 & 1,3586 \\
\hline D_9 & 5,8611 & 5,03012358 & 150,9037 & 0,01509 & 0,01526 & 101,10 & 0,7075 & & \\
\hline
\end{tabular}

A robustez do método foi comprovada, os resultados demonstram que as variações de até 2 unidades no comprimento de onda e variações da marca do espectrofotômetro não interferem no método analítico (Tabela 4).

O método proposto neste estudo para análise de suspensão oral de mebendazol apresenta como vantagens a utilização de quantidades 10 vezes menores de ácido fórmico, não tem a etapa de partição líquidolíquido, em relação ao método farmacopéico (FARMACOPÉIA BRASILEIRA, 2005) e utiliza somente etanol como solvente orgânico, que é menos agressivo ao ambiente e à saúde do analista. 


\section{CONCLUSÕES}

O método proposto neste estudo é válido para o doseamento de mebendazol matéria-prima e suspensão oral - para a formulação estabelecida - por apresentar os parâmetros de validação adequados à análise em questão, sendo uma alternativa aos métodos descritos nas monografias da Farmacopéia Brasileira 2001 e 2005.

\section{REFERÊNCIAS BIBLIOGRÁFICAS}

BRASIL. Ministério da Saúde. Agência Nacional de Vigilância Sanitária, Resolução - RE n 899, de 29 de Maio de 2003. Guia para validação de métodos analíticos e bioanaliticos. Brasília, 2003.

FARMACOPÉIA BRASILEIRA, Parte II, 3ํFascículo, 4. ed. São Paulo: Atheneu Editora, 2001

FARMACOPÉIA BRASILEIRA, Parte II, Sexto Fascículo, 4. ed. São Paulo: Atheneu Editora, 2005

GIL, E.S. Controle de qualidade fisico-químico de qualidade de medicamentos. Pharmabooks. $2^{2}$ edição. 2007

INSTITUTO NACIONAL DE METROLOGIA, NORMALIZAÇÃO E QUALIDADE INDUSTRIAL - INMETRO. DOQCGCRE-008: Orientações sobre validação de métodos de ensaios químicos. Brasil, 2003. Disponível em: http://www.inmetro.gov.br. Acesso em 10 set. 2007.

INTERNATIONAL CONFERENCE ON HARMONISATION (ICH) Q2. Validation of analytical procedures: text and methodology, 2005. Disponível em: http://www.ich.org. Acesso em 29 set. 2007

KATZUNG, B. G. Farmacologia básica e clínica. Rio de Janeiro: Editora Guanabara Koogan, 1998.

LACHMAN, L.; HANNA, S. A.; LIN, K. Controle e Garantia de Qualidade. In: LACHMAN, L.; LIEBERMAN, H. A.; KANING, J. L. Teoria e Prática na Indústria Farmacêutica. Volume II. Lisboa: Fundação Calouste Gulbenkian, 2001. Cap. 27. p.799-1104.

PINTO, T. J. A.; KANECO, T. M.; OHARA, M. T. Controle Biológico de Qualidade de Produtos Farmacêuticos, Correlatos e Cosméticos. 2 ed. São Paulo: Editora Atheneu, 2003. p. 219- 258.

SILVA, P. Farmacologia. Rio de Janeiro: Editora Guanabara Koogan, 2006

UNITED STATES. Departament of Health and Human Services. Food and Drug Administration. Reviewer Guidance: validation of chromatographic methods, 1994. Disponível em http://www.fda.gov/cder. Acesso em 02 ago.2007.

UNITED STATES PHARMACOPEIA - The National Formulary - 30th edithion, NF 25, Rockville: The United States Pharmacopeial Convention, 2007. 